ISSN:2379-1020

\title{
The Biotechnology and Genetics Race: Biomaterials Versus Biological Materials in Orthopaedic Applications
}

Murr L E*

Editorial

Department of Metallurgical \& Materials Engineering and W.M. Keck Center for 3D Innovation, The University of Texas at El Paso, El Paso, TX, USA.

\section{*Corresponding Author:}

Murr L E

Department of Metallurgical \& Materials Engineering and W.M. Keck Center for 3D Innovation, The University of Texas at El Paso, El Paso, TX, USA.

E-mail: lemurr@utep.edu

Received: May 28, 2014

Published: June 28, 2014

Citation: Murr L E (2014) The Biotechnology and Genetics Race: Biomaterials Versus Biological Materials in Orthopaedic Applications. Int J Med Biotechnol Genetics. 2(1e), 1-3. doi: http://dx.doi. org/10.19070/2379-1020-140001e

Copyright: Murr L E (C) 2014. This is an open-access article distributed under the terms of the Creative Commons Attribution License, which permits unrestricted use, distribution and reproduction in any medium, provided the original author and source are credited.

Bone consists of mature osteoblast cells and stem cells. In joints like the knee, for example, the large femur bone and the lower tibial bone are separated by cartilage and a fibro-cartilage meniscus spacer, both of which are composed of chondrocyte cells and which act as a shock absorber. These cartilage materials are often deteriorated by the accumulation of arthritic crystal aggregates and related deposits in this inter-bone region to create a variety of osteoarthritic knee problems. As these arthritic deposits build up, varying degrees of cartilage and meniscus granulation and tearing can occur. In the early stages, a minimally invasive surgical procedure called debridement or washing of the knee capsule to clean the accumulating debris along with dead chondrocycte cells can be performed. In some cases microfracture, drilling, and abrasion orthroplasty are performed to create scar-tissue to toughen the damaged cartilage. In severe circumstances of meniscus tearing it can be arthroscopically trimmed to reduce the tearing, but this can create stress displacement irregularities which can lead to further tearing and deterioration. Since the separated bones are alive, they will react to these stress variances by remodeling, which can lead to further joint deterioration, ultimately resulting in bone-on-bone situations which require surgical intervention to replace the damaged joint with implanted biomaterials. These are usually metallic alloy appliances fitted with a highly cross-linked polyethylene pad which acts as an artificial meniscus.

In total knee replacement surgery, the existing or remaining knee components are removed and replaced with a prosthetic implant appliance by first creating a skin incision down the center of the knee. Blunt dissection follows the natural cleavages between ligaments and muscles and no cutting of muscle tissue is involved. This moves the quadriceps muscle and the patella aside to expose the knee joint in a bent position. Once exposed, cutting guides and robotic devices are used to shape the end of the femur to fit the femoral appliance while the tibia is cut normal to the bone axis and a depression drilled into the cut surface. The respective prostheses are then inserted and and secured with cement such as polymethyl methacrylate (PMMA) impregnated with antibiotics to mediate infections which can cause a wide range of residual problems; the worst case involving the removal of the implants until the infection is healed, and then reinserting them [1]. Other residual problems can include the loosening of the appliances which can have a variety of consequences, including their removal and reinsertion. New approaches to implant development which include the manufacture of open cellular alloy appliances which can simulate bone structure, and engineered to have the elastic modulus or stiffness of bone to reduce or eliminate stress shielding, can allow for bone cell (osteoblast) ingrowth which eliminates cement and provides a more contiguous joint replacement [2].

But even with these new developments in more compatible, total knee replacement biomaterials technologies, the inevitable wear and related degradation elicit a finite extension of knee function which, especially in active younger people or athletes, may require at least one or more additional revision or replacement surgeries, accompanied by the prospect for related adversities described above.

In addition to biomaterials (surgical implant) solutions for cartilage remediation, autologous chrondocyte implantation (ACI) surgery is also an option where healthy cartilage is taken from an uncompromised region in the knee and chondrocyte cells are separated and cultured from it. The optimally cultured (invitro) cartilage cells are then surgically transplanted (tissue grafted) unto the degenerated knee cartilage. This procedure requires two less invasive, but nonetheless technically demanding surgeries than total knee replacement surgery, and there is also the risk that the implant will not take.

A third alternative for dealing with cartilage degeneration as well as osteoarthritis of the knee involves stem cell-based therapy; also a biological material approach. Stem cells have tremendous potential not only for treating diseased tissue by immune response modulation, but also for regeneration of the diseased tissue. They can have an anti-arthritic effect in addition to initiating cartilage tissue repair [3]. There are many hundreds of classes and sub-classes of adult stem cells that can be used in such therapies because they can differentiate into multiple cell types, including chondrocyte cells. Mesenchymal stem cells (MCSs) are pluripotent cells found in many tissues such as bone marrow aspirate and fat, and they are ideal for orthopaedic applications since they have been shown to differentiate not only into cartilage, but also bone, muscle and adipose tissue [4]. They are especially effective in articular and meniscus cartilage regeneration in human knees $[4,5]$. MSCs can also be mixed with other stem cells as well as non-stem cells or tissue 
Figure 1. Comparison of knee appearance after total knee replacement surgery (a) and autilogous stem cell cartilage replacement injection therapy (b). (a) is courtesy of Patricia Murr. (b) is courtesy of Manny Pacillas. In (b) the bracing system helps to push the femur and tibia apart slightly to allow for cartilage tissue repair
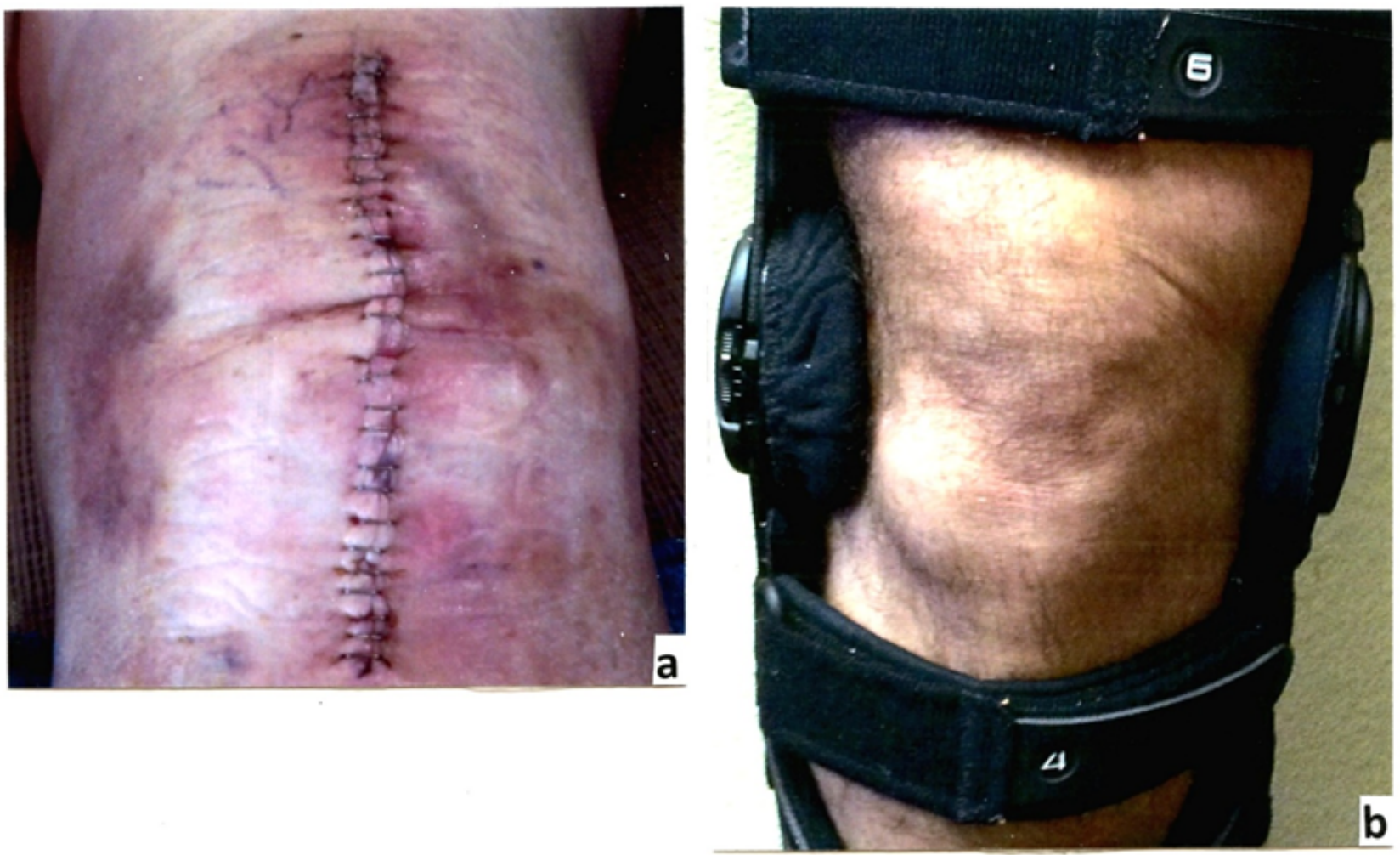

engineered scaffolds to promote healing, and genetically altered stem cells can also produce their own growth factor proteins in performing tissue repair [6].

In contrast to surgical intervention for cartilage replacement or repair, stem cell therapies involve regenerative injection routines which, although somewhat complicated with regard to creating the optimum environment for stem cell repair efficacy, is noninvasive. In addition, like cartilage transplant therapies, stem cell therapies still leave a surgical option if they are unsuccessful.

Figure 1 compares the surgically invasive total knee replacement (Fig. 1 (a)) with a typical, non-invasive regenerative injection stem cell therapy for cartilage repair and regeneration (Fig. 1 (b)) after essentially the same post-procedure time period. This comparison also implies extensive post-operative therapy associated with total knee replacement in Fig. 1 (a) in contrast to Fig. 1 (b) where there is minimal recovery from stem cell treatment, and only short-term soreness in the treated area along with some risk of bruising.

It might be noted that there are currently roughly 600,000 partial and total knee replacement surgeries performed annually in the U.S. according to The Agency for Healtcare Research and Quality. In addition, there were roughly 38,000 revision (replacement) surgeries performed in 2005, while it is estimated that under current projections for increasing replacement surgeries and increasing aging in a more active population, there will be 275,000 revision surgeries by 2030 ; a $600 \%$ increase since 2005 . In contrast, there have been no reports in the scientific literature of serious, adverse effects of stem cell therapies and procedures for either osteoarthritis or related cartilage repair and regeneration. In this context, it is estimated that the economic impact of arthritis alone in the U.S. exceeds $\$ 120$ million annually; roughly $2 \%$ of the gross domestic product (GDP).

While surgical options for cartilage repair may be improved by advances in biomaterials, including porous implants promoting bone cell ingrowth, and regimes designed to support cartilage matrix production [1,7] finding effective stem cell and genetics-related solutions for cartilage defect remediation and regeneration in orthopaedics is a more rapidly developing area of research and clinical application [8]. So the race is on between surgical interventions and biomaterials replacement in contrast to stem cell and gene-mediated stem cell injection therapies for biological material regeneration and repair, impacting a wide range of cartilage and related osteoarthritis issues. These options are contrasted in Fig 1.

\section{Conclusions}

While stem cell therapies applied to orthopaedics are still largely investigational, the U.S. orthopaedic stem cell market is projected to grow rapidly in the next few years along with more extensive clinical studies and appropriate approvals, such as FDA drugrelated approvals. It is certainly not unrealistic, viewing Fig. 1 in retrospect, that given the option, patients faced with orthopaedic joint replacement would certainly choose the non-invasive stem cell approach if covered by insurance, and with outcomes as good or better than surgical approaches. Effective stem cell procedures competitive with surgical approaches could reduce the increasing numbers of such procedures and correspondingly reduce the number of revision surgeries, indicated previously to be projected 
at current rates to increase dramatically in the next 15 years.

\section{References}

[1]. Murr LE (2003) Some comments on orthopaedic implant infection: Biomaterials issues (Editorial). J Biotechnol Biomater 3 (3) : 100-119.

[2]. Murr LE, Gaytan SM, Martinez E, Medina F, Wicker RB (2012) Next generation orthopedic implants by additive manufacturing using electron beam melting. Int J Biomater 245727.

[3]. Luyten FP (2004) Mesenchymal stem cells in osteoarthritis. Current Opinion in Rheumatology 16 (5) : 599-603.

[4]. Caplan A (2010) Mesenchymal stem cells : The past, the present, the future. Cartilage 1 (1) : 6-9.
[5]. Diekman B, Guilak F (2013) Stem-cell based therapies for osteoarthritis : Challenges and opportunities. Current Opinion in Rheumatology 25 (1) : 119-126.

[6]. Brunger JM, Huynh NPT, Guenther CM, Perez-Pinera P, Moutos FT, et al. (2014) Scaffold-mediated lentivival transduction for functional tissue engineering of cartilage. Proc Matl Acad Sci (USA). D01 : 1073/pnas. 1321744111 - 1DC Supplemental.

[7]. Sharma B, Femanian B, Gibson M, Untersnan ., Herzka DA, et al. (2013) Human cartilage repair with a photoactive adhesive-hydrogel composite. Sci Transl Med 5: 167.

[8]. Maclaine SE, McNaunara LE, Bennett AJ, Dalby MJ, Meck RM (2013) Developments in stem cells : Implications for future joint replacements. Proc Inst Mech.Eng H. Mar. 227 (3) : 275-283. 